\title{
Pseudomonas aeruginosa in the ICU: prevalence, resistance profile, and antimicrobial consumption
}

\author{
Ághata Cardoso da Silva Ribeiro ${ }^{[1]}$, Márcia Terezinha Lonardoni Crozatti ${ }^{[1]}$, \\ Adilson Aderito da Silva ${ }^{[2],}$ Rodrigo Spineli Macedo ${ }^{[3]}$, \\ Antonia Maria de Oliveira Machado ${ }^{[4]}$ \\ and Antonio Távora de Albuquerque Silva ${ }^{[1]}$
}

[1]. Universidade Federal de São Paulo, Instituto de Ciências Ambientais, Químicas e Farmacêuticas, Diadema, SP, Brasil.

[2]. Universidade Presbiteriana Mackenzie, Centro de Ciências Sociais e Aplicadas, São Paulo, SP, Brasil.

[3]. Universidade Federal de São Paulo, Hospital Universitário da UNIFESP/Hospital São Paulo, São Paulo, SP, Brasil.

[4]. Universidade Federal de São Paulo. Departamento de Medicina. Laboratório Central do Hospital São Paulo, São Paulo, SP, Brasil.

\begin{abstract}
Introduction: Pseudomonas aeruginosa is one of the main pathogens causing infection in intensive care units (ICUs) and usually presents antimicrobial resistance. Methods: Data were obtained from ICUs between 2010 and 2013. Results: P. aeruginosa had a prevalence of $14.5 \%$ of which $48.7 \%$ were multidrug resistant. We observed increasing resistance to carbapenems and polymyxin $\mathrm{B}$ and growing consumption of aminoglycosides, meropenem, ceftazidime, and polymyxin B. The regression impact between resistance and consumption was significant with respect to amikacin, imipenem, meropenem, and polymyxin B. Conclusions: Monitoring antimicrobial consumption and resistant microorganisms should be reinforced to combat antimicrobial- and multidrug resistance.
\end{abstract}

Keywords: Pseudomonas aeruginosa. Antimicrobial agents. Drug resistance.

Antimicrobial resistance is a public health concern ${ }^{1}$. Among resistant pathogens, the bacterium Pseudomonas aeruginosa is of note because it is often associated with high rates of morbidity and mortality in patients in intensive care units (ICUs) ${ }^{2}$. P. aeruginosa is resistant to several antibiotics and has the ability to rapidly develop resistance to new antimicrobials. Moreover, $P$. aeruginosa was the first bacterium to present multidrug-resistant (MDR) phenotypes ${ }^{3}$.

Therefore, it is important to monitor the consumption of antibiotics, especially in a hospital environment, to avoid the development of resistance and improve the therapeutic efficacy of these drugs against $P$. aeruginosa.

To address the above issues, this study aimed to describe the prevalence of resistant and MDR isolates of $P$. aeruginosa in nine ICUs of a university hospital, as well as to describe

Corresponding author: Ághata Cardoso da Silva Ribeiro.

e-mail: aghata.cardoso@unifesp.br

Orcid: 0000-0002-4997-0505

Received 22 November 2018

Accepted 11 July 2019 the consumption of antimicrobial agents using a defined daily dose (DDD) and investigate the relationship between the consumption of and resistance to antibiotics in the nine ICUs.

This was a retrospective descriptive study, conducted from January 2010 to December 2013 in ICUs of the Hospital São Paulo - university hospital (HSP-HU/Unifesp) in the city of São Paulo (Brazil).

For resistance and susceptibility analysis, data from the Clinical Laboratory of the hospital were used. Samples were obtained from patients hospitalized in at least one of the ICUs selected during the study period.

P. aeruginosa isolates were considered to be MDR if they showed resistance to three or more classes of antimicrobial agents, with resistance to at least one antibiotic in each class ${ }^{4}$.

Data on the consumption of antimicrobials (in grams) were provided by the Supplies System of the hospital, and the hospitalization rate in the study period was provided by the Hospital Statistics Sector. Antimicrobial agents were classified 
according to the Anatomical Therapeutic Chemical system, and consumption was measured in DDD per 100 bed-days ${ }^{5}$.

Susceptibility tests performed on samples from anal and rectal swabs for Klebsiella pneumoniae carbapenemase surveillance and vancomycin-resistant enterococci surveillance were excluded from the study.

For the organization and analysis of the data, Microsoft ${ }^{\circledR}$ Office Excel for Mac, version 16.27 (Microsoft Corporation) and the Statistical Package for Social Sciences version 20 (IBM Corp, Chicago, IL, USA) were used.

The resistance trend of $P$. aeruginosa, monthly consumption of antimicrobial agents, and the association between antimicrobial agent consumption and resistance of $P$. aeruginosa were analyzed using a linear regression model. The $\beta$ coefficient indicates the association between a dependent variable and independent variables analyzed by the linear regression model. The significance level was set at 0.05 .

The remaining analyses were for normality, linearity, and homoscedasticity. For normality analyses, the KolmogorovSmirnov (KS) test (significance $>0.05$ ) was performed and all the variables obtained were considered normal, except for the data on consumption of ceftazidime and polymyxin resistance. The homoscedasticity and linearity analyses were conducted by graphical analyses. Homoscedasticity was analyzed using the standardized variables against the standardized predicted values.

This study is part of the project "Prevalent microorganisms in ICU: antimicrobial agents consumption and resistance profiles," approved by the Research Ethics Committee (opinion 921.500), in accordance with Resolution No. 466 of December 12, 2012, of the National Health Council.

Of 6,473 microorganisms isolated, 939 were identified as P. aeruginosa (14.5\%). P. aeruginosa was the second most prevalent bacterium during the study period, after Acinetobacter baumannii (17.3\%). The prevalence of $P$. aeruginosa isolates resistant to antimicrobial agents by year is presented in Table 1.

Increasing resistance of $P$. aeruginosa to imipenem $(\beta=0.419 ; p=0.003)$, meropenem $(\beta=0.485 ; p=0.000)$, and polymyxin $B(\beta=0.401, p=0.005)$ was statistically significant. The prevalence of resistance to imipenem was $50 \%$ in January 2010 and $71.4 \%$ in December 2013; that of resistance to meropenem was $44.4 \%$ in January 2010 and $71.4 \%$ in December 2013, while the prevalence of resistance to polymyxin B was zero in January 2010 and 7.1\% in December 2013. However, significantly decreasing resistance of $P$. aeruginosa to amikacin $(\beta=-0.345 ; p=0.016)$ and piperacillin/tazobactam $(\beta=-0.301$, $\mathrm{p}=0.038$ ) were observed (Table 2).

It is noteworthy that $457(48.7 \%)$ of the 939 isolates of P. aeruginosa were classified as MDR P. aeruginosa (PaMDR), with a higher prevalence in the years 2010 (51.2\%) and 2013 $(52.9 \%)$.

Consumption of amikacin $(\beta=0.793 ; p=0.000)$, gentamicin ( $\beta=0.746 ; p=0.000)$, meropenem $(\beta=0.571 ; p=0.000)$, ceftazidime $(\beta=0.336 ; \mathrm{p}=0.020)$, and polymyxin $\mathrm{B}(\beta=0.625$; $\mathrm{p}=0.000$ ) were significantly increasing. In contrast, consumption of imipenem $(\beta=-0.875 ; p=0.000)$ and cefepime $(\beta=-0.825$; $\mathrm{p}=0.000$ ) were significantly decreasing (Table 2).

In addition, significant associations were observed between the consumption of and resistance of $P$. aeruginosa to amikacin $(\beta=-0.361 ; \mathrm{p}=0.012)$, imipenem $(\beta=-0.316 ; \mathrm{p}=0.029)$, meropenem $(\beta=0.327 ; \mathrm{p}=0.023)$, and polymyxin $\mathrm{B}(\beta=0.351$; $\mathrm{p}=0.014)($ Table 3).

In this study, P. aeruginosa was the second most frequently isolated bacterium, representing $14.5 \%$ of the total isolates, which demonstrates the clinical importance of this microorganism. The results obtained are consistent with those of other studies, such as the one performed in ICUs of a public hospital in Ceará (Brazil), wherein P. aeruginosa represented $33.8 \%$ of the isolates ${ }^{6}$, and that performed in an ICU of Anesthesiology in Turkey, wherein the prevalence of $P$. aeruginosa was $14.29 \%{ }^{2}$, demonstrating that the high prevalence of this microorganism is common in various countries.

The resistance profiles of $P$. aeruginosa against the tested antibiotics are presented in Table 1. It is noteworthy that Biswall et al. (2014), in India, reported similar resistance prevalence results for aztreonam (41.38\%) and piperacillin/tazobactam $(34.5 \%)$, as well as zero resistance to colistin (or polymyxin E). However, the results were different for amikacin (81.03\%), gentamicin (81.03\%), ceftazidime (70.68\%), imipenem (18.9\%), and meropenem $(13.79 \%)^{7}$. Based on these data, there may be variations in microbial resistance profiles depending on the study site, which shows the importance of determining resistance profiles to be able to select the most appropriate antimicrobial therapy.

It is also worth noting that the prevalence of resistance of $P$. aeruginosa to imipenem and meropenem showed an increasing trend in the study period. These data concur with the results of a study carried out at a hospital in the state of Santa Catarina (Brazil), which indicated that resistance to carbapenems was increasing. Thus, in 2004, the resistance of P. aeruginosa to imipenem was $6.06 \%$, increasing to $48.09 \%$ in 2008 ; for meropenem, the resistance increased from $6.89 \%$ in 2004 to approximately $20 \%$ in 2008. Similarly, Xu et al. (2013) in a study performed at a hospital in China found increasing resistance to imipenem $(\beta=4.620 ; p<0.001)$ and meropenem $(\beta=4.624 ; p<0.001)^{9}$. Although carbapenems are one of the most potent classes of antimicrobials against $P$. aeruginosa, increasing resistance to these drugs is an endemic problem in several countries; therefore, the World Health Organization (WHO) considers the development of new drugs for this microorganism a priority ${ }^{10}$.

In addition, the present study showed a decreasing prevalence of resistance of $P$. aeruginosa to amikacin and piperacillin/tazobactam, which differs from the results of $\mathrm{Xu}$ et al. (2013), who showed that resistance to amikacin $(\beta=0.488$; $\mathrm{p}=0.332)$ and piperacillin/tazobactam $(\beta=4.346 ; \mathrm{p}<0.001)$ was increasing.

Although the resistance of $P$. aeruginosa to polymyxin B was found to be low $(0.9 \%)$ in the present study, resistance to 
TABLE 1: Prevalence of $P$. aeruginosa isolates resistant to the tested antibiotics, based on laboratory findings of patients in nine ICUs from January 2010 to December 2013.

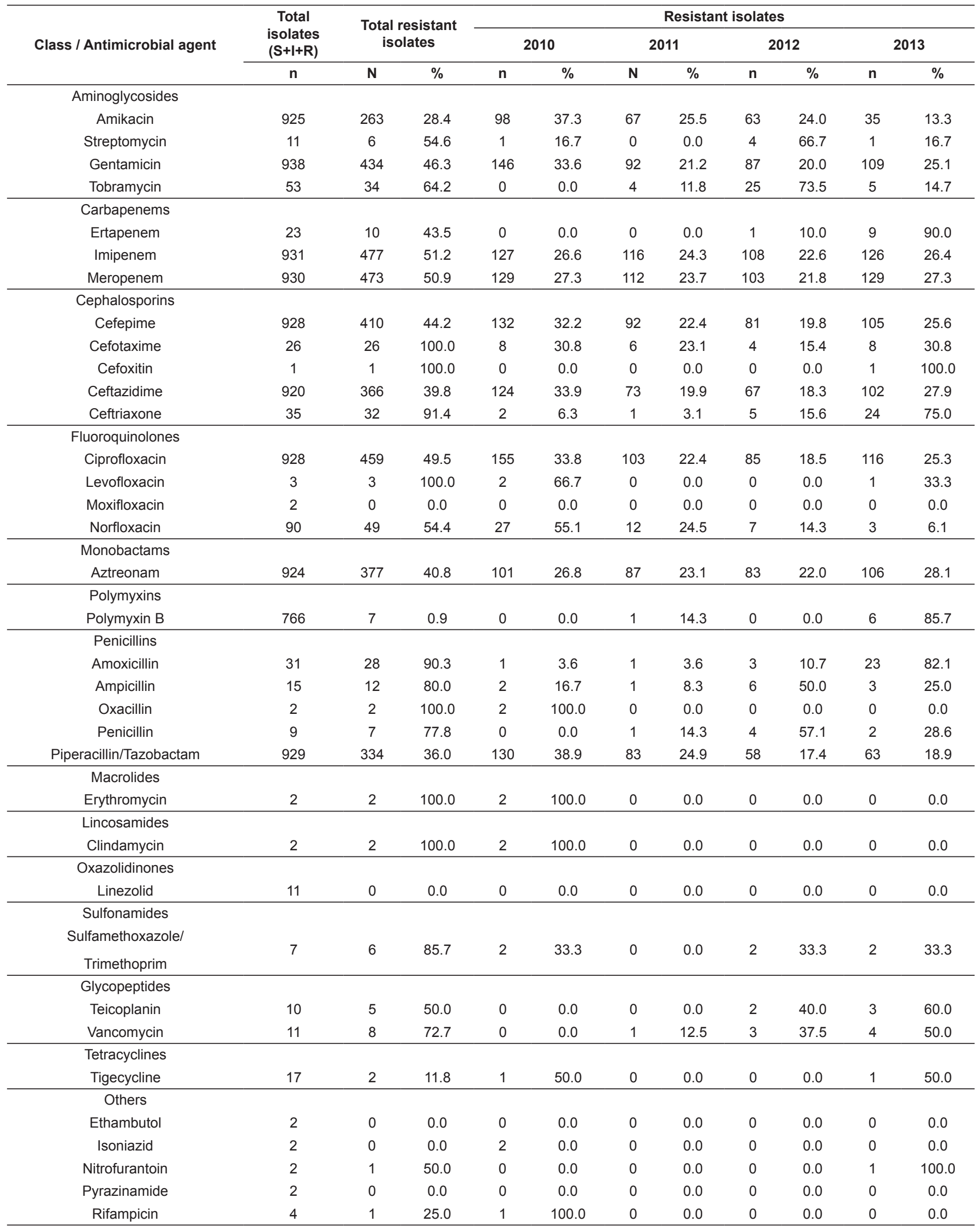

I: intermediate; R: resistant; S: susceptible. 
TABLE 2: Trends in the prevalence of resistance of $P$. aeruginosa to and consumption of antimicrobial agents of clinical interest in nine ICUs from January 2010 to December 2013.

Trend in the prevalence of $P$. aeruginosa resistance

Trend in antimicrobial agent consumption

\begin{tabular}{|c|c|c|c|c|c|c|c|c|}
\hline \multirow{2}{*}{$\begin{array}{c}\text { Antimicrobial } \\
\text { agent }\end{array}$} & & & \multirow[b]{2}{*}{ Trend } & \multirow[b]{2}{*}{$\begin{array}{c}\text { KS } \\
\text { significance }\end{array}$} & & & & \\
\hline & $\beta$ & $\mathbf{p}$ & & & $\beta$ & $\mathbf{p}$ & Trend & $\begin{array}{c}\text { KS } \\
\text { significance }\end{array}$ \\
\hline Amikacin & -0.345 & 0.016 & decreasing & 0.974 & 0.793 & 0.000 & increasing & 0.445 \\
\hline Gentamicin & 0.037 & 0.802 & increasing & 0.769 & 0.746 & 0.000 & increasing & 0.845 \\
\hline Ciprofloxacin & 0.040 & 0.786 & increasing & 0.966 & 0.188 & 0.200 & increasing & 0.341 \\
\hline Imipenem & 0.419 & 0.003 & increasing & 0.927 & -0.875 & 0.000 & decreasing & 0.904 \\
\hline Meropenem & 0.485 & 0.000 & increasing & 0.754 & 0.571 & 0.000 & increasing & 0.301 \\
\hline Cefepime & 0.163 & 0.269 & increasing & 0.470 & -0.825 & 0.000 & decreasing & 0.575 \\
\hline Ceftazidime & 0.217 & 0.138 & increasing & 0.967 & 0,336 & 0.020 & increasing & 0.016 \\
\hline $\begin{array}{l}\text { Piperacillin/ } \\
\text { Tazobactam }\end{array}$ & -0.301 & 0.038 & decreasing & 0.673 & 0.245 & 0.094 & increasing & 0.889 \\
\hline Polymyxin B & 0.401 & 0.005 & increasing & 0.010 & 0.625 & 0.000 & increasing & 0.210 \\
\hline
\end{tabular}

$\beta$ coefficient: indicates the association between a dependent variable and independent variables by a linear regression model. The significance level was set at 0.05 . KS significance: indicates the significance of the Kolmogorov-Smirnov normality test. Variables were considered normal when KS $>0.005$.

TABLE 3: Association between the consumption of antimicrobial agents of clinical interest and the prevalence of $P$. aeruginosa resistance in nine ICUs from January 2010 to December 2013.

\begin{tabular}{|c|c|c|c|c|}
\hline Antimicrobial agent & $\beta$ & p & Impact & $\begin{array}{c}\text { KS } \\
\text { significance }\end{array}$ \\
\hline Amikacin & -0.361 & 0.012 & Negative & 0.774 \\
\hline Gentamicin & 0.082 & 0.579 & Positive & 0.858 \\
\hline Imipenem & -0.316 & 0.029 & Negative & 0.350 \\
\hline Meropenem & 0.327 & 0.023 & Positive & 0.534 \\
\hline $\begin{array}{l}\text { Piperacillin/ } \\
\text { Tazobactam }\end{array}$ & -0.062 & 0.675 & Negative & 0.953 \\
\hline Polymyxin B & 0.351 & 0.014 & Positive & 0.001 \\
\hline
\end{tabular}

$\beta$ coefficient: indicates the association between a dependent variable and independent variables by a linear regression model. The significance level was set at 0.05 . KS significance: indicates the significance of the Kolmogorov-Smirnov normality test. Variables were considered normal when KS $>0.005$. 
this antibiotic was observed to increase $(\beta=0.401, \mathrm{p}=0.005)$. This result is worth mentioning as polymyxins are considered the last line of defense against gram-negative bacteria and have been classified by the WHO as critically important for human medicine ${ }^{11}$.

Among the isolates of $P$. aeruginosa, $48.7 \%$ were classified as MDR. This result is similar to the prevalence of $30.5 \%$ observed in a multicenter study of patients admitted to hospitals in Europe and the United States ${ }^{4}$. Similarly, a study carried out at a Spanish hospital indicated that $33.3 \%$ of $P$. aeruginosa isolates were classified as $\mathrm{MDR}^{12}$. It is also worth noting that in Brazil, Neves et al. (2010) reported a frequency of $23.1 \%$ of PaMDR, in a study carried out at the Clinics Hospital of Botucatu of the School of Medicine of the State of São Paulo ${ }^{13}$.

Therefore, it is important to note that the treatment options for PaMDR are limited. Most PaMDR are susceptible to polymyxins (polymyxin B and colistin). Aminoglycosides may be another alternative ${ }^{3}$.

Considering the consumption of antimicrobial agents, increasing tendency for the consumption of amikacin and gentamicin was observed in the present study. However, Vega et al. (2015) observed a decrease in the consumption (in DDD/100 patients-day) of amikacin (from 0.87 in 2008 to 0.31 in 2011) and an increase in the consumption of gentamicin (from 1.17 in 2008 to 10.67 in 2011) in a study carried out in an adult ICU in Argentina ${ }^{14}$. In another study, an increase in the consumption (in DDD/100 patients-days) of both amikacin (from 1.48 in 1999 to 1.91 in 2008) and gentamicin (from 5.02 in 1999 to 6.88 in 2008) was observed in ICUs in the Czech Republic ${ }^{15}$.

In the present study, a downward trend in imipenem consumption was identified, while meropenem consumption was increasing. In the study by Vojtová et al. (2011), increasing consumption of both imipenem (from 0.33 in 1999 to 1.60 in 2008) and meropenem (from 0.88 in 1999 to 3.56 in 2008) was observed $^{15}$.

In addition, a decreasing trend in the consumption of cefepime was observed in the present study, whereas ceftazidime showed an increasing trend. In the study by Vega et al. (2015), the consumption (in DDD/100 beds-day) of cefepime and ceftazidime decreased between 2008 and 2011 from 10.19 to 1.48 and 2.2 to 0.25 , respectively ${ }^{14}$.

Furthermore, a growing trend of consumption was found for polymyxin B, perhaps because of the increased prevalence of PaMDR in the study period and because polymyxins are currently the last option for monotherapy against resistant and $\mathrm{PaMDR}^{3}$, and are therefore extensively used for this purpose.

By analyzing the correlation between the prevalence of resistance to and the consumption of antimicrobial agents, we observed a negative association between the consumption of amikacin and imipenem and prevalence of $P$. aeruginosa resistance to these antibiotics. However, even though the consumption of amikacin increased during the study period, we cannot conclude that the decrease in the prevalence of resistance was only due to the possible effectiveness of the antimicrobial since it is known that the decrease in prevalence of bacterial resistance also depends on other factors ${ }^{1}$ such as increased surveillance, campaigns to combat the spread of resistance, and changes in microbial resistance profiles.

It is still noteworthy that a positive impact and association between the consumption of meropenem and polymyxin B and the prevalence of resistance of $P$. aeruginosa to these antibiotics were detected. Xu et al. (2013) obtained similar results for meropenem $(\beta=1.241 ; \mathrm{p}<0.001)$ but different results for imipenem $(\beta=1.238 ; p<0.001)^{9}$. As for polymyxin $B$, the result obtained is worrisome as polymyxins are the last option for monotherapy against resistant and $\mathrm{PaMDR}^{3}$. However, it is important to note that in the present study, P. aeruginosa showed a low resistance profile to polymyxin $\mathrm{B}$.

The present study has the following limitations: (i) the presented consumption data were general data for the nine selected ICUs, not specific data for patients with P. aeruginosa infection; and (ii) other factors that influence resistance were not considered, although antimicrobial consumption is one of the most important factors ${ }^{1}$.

Thus, a prospective study using specific antimicrobial consumption data and data from each patient with $P$. aeruginosa infection, as well as the analysis of all associated factors and/or resistance influencers, may help better establish the correlations between bacterial resistance and antimicrobial consumption, as well as between bacterial resistance and other associated factors and/or resistance influencers.

In the present study, we determined the prevalence of resistant and MDR isolates of $P$. aeruginosa, as well as the levels of antibiotic consumption in ICUs of a university hospital in São Paulo (Brazil). The results of this work emphasize the importance of monitoring the consumption of antimicrobial agents and combating the increase of microbial resistance, including multidrug resistance.

\section{ACKNOWLEDGMENTS}

We express our gratitude to Universidade Federal de São Paulo (UNIFESP) which supported this work by providing the data of the study population.

\section{Conflict of Interest}

The authors declare that there are no conflicts of interest.

\section{REFERENCES}

1. World Health Organization (WHO). Antimicrobial Resistance. Global Report on surveillance [Internet]. 1th ed. Geneva: WHO Press; 2014 Apr [cited 2018 Nov 22]. 256p. Available from: http:// apps.who.int/iris/bitstream/handle/10665/112642/9789241564748_ eng.pdf?sequence $=1$

2. Dereli N, Ozayar E, Degerli S, Sahin S, Koç F. Three-Year Evaluation of Nosocomial Infection Rates of the ICU. Braz J Anesthesiol. 2013;63(1):73-8.

3. Fraimow HS, Tsigrelis C. Antimicrobial Resistance in the Intensive Care Unit: Mechanisms, Epidemiology, and management of Specific Resistant Pathogens. Crit Care Clin. 2011;27(1):163-205. 
4. Micek ST, Wunderink RG, Kollef MH, Chen C, Rello J, Chastre J et al. An international multicenter retrospective study of Pseudomonas aeruginosa nosocomial pneumonia: impact of multidrug resistance. Crit Care. 2015;19:219-27.

5. World Health Organization Collaborating Centre for Drug Statistics Methodology - WHOCC [Internet]. Norway: Norwegian Institute of Public Health; 2015 [updated 2017 Dec 20; cited 2018 Nov 22]. Available from: http://www.whocc.no/atc_ddd_index/

6. Barros LM, Bento JNC, Caetano JA, Moreira RAN, Pereira FGF, Frota NM et al. Prevalência de micro-organismos e sensibilidade antimicrobiana de infecções hospitalares em unidade de terapia intensiva de hospital público no Brasil. Rev Ciênc Farm Básica e Apl. 2012;33(3):429-35.

7. Biswall I, Arora BS, Kasana D, Neestushree. Incidence of Multidrug Resistant Pseudomonas aeruginosa Isolated from Burn Patients and Environment of Teaching Institution. J Clin Diagn Res. 2014;8(5):26-9.

8. Baumgart AMK, Molinari MA, Silveira ACO. Prevalence of carbapenem resistant Pseudomonas aeruginosa and Acinetobacter Baumannii in high complexity hospital. Braz J Infect Dis. 2010;14(5):433-6.

9. Xu J, Duan X, Wu H, Zhou Q. Surveillance and Correlation of Antimicrobial Usage and Resistance of Pseudomonas aeruginosa: A Hospital Population-Based Study. PLoS ONE [Internet]. 2013 Nov 8 [cited 2018 Nov 22]; 8(11):e78604. Available from: https:// www.ncbi.nlm.nih.gov/pmc/articles/PMC3826718/
10. World Health Organization (WHO). Critically important antimicrobials for human medicine [Internet]. 5rd ed. Geneva: World Health Organization; 2016; June [cited 2018 Nov 22]. 41p. Available from: http://apps.who.int/iris/bitstream/hand le/10665/255027/9789241512220-eng.pdf;jsessionid=4CA3754731 D70DB15F9C963004DE86F0?sequence $=1$

11. World Health Organization (WHO). Global priority list of antibioticresistant bacteria to guide research, discovery, and development of new antibiotics [Internet]. Geneva: WHO; 2016 [cited 2018 Nov 20]. 7p. Available from: http://www.who.int/medicines/publications/ WHO-PPL-Short_Summary_25Feb-ET_NM_WHO.pdf?ua=1

12. Morales E, Cots F, Sala M, Comas M, Belvis F, Riu M et al. Hospital costs of nosocomial multi-drug resistant $P$ seudomonas aeruginosa acquisition. BMC Health Serv Res. 2012;12(122):1-8.

13. Neves MT, Lorenzo MEP, Almeida RAMB, Fortaleza CMB. Antimicrobial use and incidence of multidrug-resistant Pseudomonas aeruginosa in a teaching hospital: an ecological approach. Rev Soc Bras Med Trop. 2010;43(6):629-32.

14. Vega EM, Fontana D, Iturrieta M, Segovia L, Rodríguez G, Agüero S. Consumo de antimicrobianos en la Unidad de Terapia Intensiva del Hospital Dr. Guillermo Rawson-San Juan, Argentina. Rev Chilena Infectol. 2015;32(3):259-65.

15. Vojtová V, Kolár M, Hricová K, Uvízl R, Neiser J, Blahut L et al. Antibiotic utilization and Pseudomonas aeruginosa resistance in intensive care units. New Microbiol. 2011;34(3):291-8. 